\title{
PRODUTIVIDADE DA CULTURA DA MELANCIA EM FUNÇÃO DE FONTES E DOSES DE POTÁSSIO
}

\author{
Yield of watermelon fruits as a function of sources and doses of potassium
}

\author{
Arthur Bernardes Cecílio Filho ${ }^{1}$, Leilson Costa Grangeiro ${ }^{1}$
}

\begin{abstract}
RESUMO
Foram conduzidos dois experimentos em propriedades rurais próximas à cidade de Borborema - SP, nos períodos de outubro a dezembro de 2001 e de fevereiro a abril de 2002, com o objetivo de avaliar a produtividade de melancia (Citrullus lanatus (Thumb.) Matsum. \& Nakai), híbrido Tide, em função de fontes e doses de potássio. O delineamento experimental utilizado foi o de blocos casualizados completos, em esquema fatorial $3 \times 4$, com três repetições, sendo utilizadas as fontes: cloreto K$\mathrm{Cl})$, nitrato $\left(\mathrm{KNO}_{3}\right)$ e sulfato de potássio $\left(\mathrm{K}_{2} \mathrm{SO}_{4}\right)$ e as doses: 50; 100; 200 e $300 \mathrm{~kg} \mathrm{de} \mathrm{K}_{2} \mathrm{O}$ ha $^{-1}$. Foram avaliados o peso médio de fruto, o número de frutos e produção por planta e produtividade. O maior número de frutos por planta estimado foi obtido nas doses de 190 e $300 \mathrm{~kg} \mathrm{~K}_{2} \mathrm{O}$ ha ${ }^{-1}$, respectivamente, nas fontes $\mathrm{KNO}_{3}$ e $\mathrm{KCl}$. Não houve diferença significativa entre as doses para $\mathrm{K}_{2} \mathrm{SO}_{4}$. Para o peso médio de fruto, houve diferença significativa entre as fontes apenas no cultivo de fevereiro a abril de 2002 , quando o $\mathrm{KCl}$ proporcionou maior peso. A maior produção por planta foi obtida com $\mathrm{K}_{2} \mathrm{SO}_{4}$, seguido por $\mathrm{KNO}_{3}$ e $\mathrm{KCl}$. Entretanto, as doses de potássio para as máximas produtividades foram de 132, 193 e $205 \mathrm{~kg} \mathrm{~K}_{2} \mathrm{O} \mathrm{ha}^{-1}$, respectivamente, para as fontes $\mathrm{K}_{2} \mathrm{SO}_{4}, \mathrm{KNO}_{3}$ e $\mathrm{KCl}$. O plantio de outubro a dezembro de 2001 proporcionou maior peso médio, produção de frutos por planta e produtividade, ao passo que o maior número de frutos por planta foi obtido no cultivo de fevereiro a abril de 2002.
\end{abstract}

Termos para indexação: Citrullus lanatus, nutrição de plantas, fertilizantes potássicos.

\begin{abstract}
Two field experiments were conducted in Borborema, State of São Paulo, from periods of October to December of the 2001 and February to April of 2002, with the objective of evaluate the yield of watermelon (Tide hybrid) (Citrullus lanatus (Thumb.) Matsum. \& Nakai) in function of sources and doses of potassium. The experimental design was a randomized complete block, with three replications, in a 3 x 4 factorial scheme, being evaluated sources: potassium chloride, nitrate and sulphate and doses: 50,100, 200 and $300 \mathrm{~kg} \mathrm{~K}_{2} \mathrm{O} \mathrm{ha}^{-1}$. The average weight of fruits, number of fruits and production per plant and yield were evaluated. The biggest number of fruits for plant was achieved with 190 and $300 \mathrm{~K}_{2} \mathrm{O} \mathrm{ha}^{-1}$ of $\mathrm{KNO}_{3}$ and $\mathrm{KCl}$, respectively. The weight of fruit differed among sources only in the second experiment, where $\mathrm{KCl}$ provided greater weight. Maximum yield were obtained with doses of 132, 193 e $205 \mathrm{~kg} \mathrm{~K}_{2} \mathrm{O} \mathrm{ha}{ }^{-1}$, respectively from $\mathrm{K}_{2} \mathrm{SO}_{4}, \mathrm{KNO}_{3}$ e $\mathrm{KCl}$. In the experiment from October to December of 2001, were obtained the greatest average weight, production of fruits per plant and yield, while the biggest number of fruits per plant was achieved in the experiment of February to April of 2002.
\end{abstract}

Index terms: Citrullus lanatus, plant nutrition, potassium fertilizers.

(Recebido para publicação em 23 de outubro de 2003 e aprovado em 5 de janeiro de 2004)

\section{INTRODUÇÃO}

O potássio desempenha muitas funções na bioquímica e fisiologia da planta, destacando-se sua participação nos processos de fotossíntese, transporte e armazenamento de assimilados. É requerido em grande quantidade pelas culturas, sendo o cátion mais abundante nos vegetais (MALAVOLTA e CROCOMO, 1982; MENGEL e KIRKBY, 1987; MARSCHNER, 1995). Segundo Faquin (1994), o requerimento de potássio para o ótimo desenvolvimento das plantas é de aproximadamente 20 a $50 \mathrm{~g} \mathrm{~kg}^{-1}$ de massa seca, variando conforme a espécie, a época e o órgão analisado.

A exigência de potássio pela cultura da melancia (Citrullus lanatus (Thumb.) Matsum. \& Nakai) é superior à de nitrogênio, sendo exigido em maior proporção após a frutificação. Em trabalho desenvolvido por Grangeiro e Cecílio Filho (2002), o potássio foi o nutriente mais absorvido pelo híbrido de melancia Tide, com maior acúmulo no período de 60 a 75 dias após transplantio. Do total extraído pela cultura, $24 \%$ encontravam-se na parte vegetativa e $76 \%$ nos frutos, inferindo-se, com isso, que a exportação de potássio pela 
melancieira é elevada. Esta informação auxilia na compreensão dos resultados obtidos por diversos autores, os quais obtiveram incrementos significativos na produção de melancia com a adubação potássica (SUNDSTROM e CARTER, 1983; DESWAL e PATIL, 1984; ZENG e JIANG, 1988; SIMONNE et al., 1992; LOCASCIO e HOCHMUTH, 2002).

Os principais fertilizantes potássicos são o cloreto, o nitrato e o sulfato de potássio, porém, devido ao íon acompanhante, os efeitos que proporcionam no crescimento e na produção podem ser distintos. A escolha de uma dessas fontes deve ser baseada na necessidade da cultura, no método de aplicação, no preço e na disponibilidade para aquisição no mercado. Na cultura da melancia, o cloreto de potássio é a fonte mais utilizada por causa do menor preço. Entretanto, não existem pesquisas que comprovem a maior eficiência dessa fonte para a cultura. Para algumas hortaliças, como batata e berinjela, a utilização de $\mathrm{KCl}$ tem proporcionado rendimentos inferiores em relação ao sulfato de potássio (PANIQUE et al., 1997; WUZHONG, 2002). Em tomate, Locascio et al. (1997) verificaram diferença significativa entre fontes de $\mathrm{K}$ em apenas um entre nove experimentos realizados, no qual o nitrato de potássio proporcionou um rendimento $19 \%$ superior ao $\mathrm{KCl}$. No meloeiro, não houve diferença entre as fontes cloreto de potássio, sulfato de potássio e sulfato de potássio e magnésio (MAYNARD, 2002).

Com o presente trabalho objetivou-se avaliar a produtividade de frutos de melancia em função de fontes e doses de potássio.

\section{MATERIAL E MÉTODOS}

Foram conduzidos dois experimentos em propriedades rurais próximas à cidade de Borborema - SP, nos períodos de outubro a dezembro de 2001 e de fevereiro a abril de 2002, cujas coordenadas geográficas são $49^{\circ}$ 5' de longitude, $21^{\circ} 37^{\prime}$ de latitude e $429 \mathrm{~m}$ de altitude. Os solos das áreas experimentais foram classificados como Argissolo Vermelho-Amarelo distrófico textura média (EMBRAPA, 1999). Para a caracterização dos solos, foram coletadas amostras compostas na profundidade de 0 a $20 \mathrm{~cm}$, cujas análises químicas (RAIJ et al., 1996) revelaram os resultados: área 1 (cultivo de out. a dez. de 2001): $\mathrm{pH}\left(\mathrm{CaCl}_{2}\right)=4,2 ; \mathrm{M} . \mathrm{O} .=11 \mathrm{~g} \mathrm{dm}^{-3} ; \mathrm{P}(\mathrm{re}-$ sina) $=2 \mathrm{mg} \mathrm{dm}^{-3} ; \mathrm{S}=3 \mathrm{mg} \mathrm{dm}^{-3} ; \mathrm{K}=1,1 \mathrm{mmol}_{\mathrm{c}} \mathrm{dm}^{-3}$; $\mathrm{Ca}=7 \mathrm{mmol}_{\mathrm{c}} \mathrm{dm}^{-3} ; \mathrm{Mg}=3 \mathrm{mmol}_{\mathrm{c}} \mathrm{dm}^{-3} ; \mathrm{H}+\mathrm{Al}=28$ $\mathrm{mmol}_{\mathrm{c}} \mathrm{dm}^{-3} ; \mathrm{SB}=11 \mathrm{mmol}_{\mathrm{c}} \mathrm{dm}^{-3} ; \mathrm{T}=39$ e V=28\% e área 2 (cultivo de fev. a abr. de 2002): $\mathrm{pH}\left(\mathrm{CaCl}_{2}\right)=4,8$; M.O. $=19 \mathrm{~g} \mathrm{dm}^{-3} ; \mathrm{P}$ (resina) $=3 \mathrm{mg} \mathrm{dm}^{-3} ; \mathrm{S}=5 \mathrm{mg} \mathrm{dm}^{-3}$;
$\mathrm{K}=1,0 \mathrm{mmol}_{\mathrm{c}} \mathrm{dm}^{-3} ; \mathrm{Ca}=9 \mathrm{mmol}_{\mathrm{c}} \mathrm{dm}^{-3} ; \mathrm{Mg}=5 \mathrm{mmol}_{\mathrm{c}}$ $\mathrm{dm}^{-3} ; \mathrm{H}+\mathrm{Al}=16 \mathrm{mmol}_{\mathrm{c}} \mathrm{dm}^{-3} ; \mathrm{SB}=15,0 \mathrm{mmol}_{\mathrm{c}} \mathrm{dm}^{-3} ; \mathrm{T}$ $=31 \mathrm{e} \mathrm{V}=48 \%$.

$\mathrm{O}$ delineamento experimental utilizado foi o de blocos casualizados completos, em esquema fatorial $3 \mathrm{x}$ 4, com três repetições, sendo avaliadas as fontes cloreto, nitrato e sulfato de potássio e as doses 50; 100; 200 e $300 \mathrm{~kg}$ de $\mathrm{K}_{2} \mathrm{O} \mathrm{ha}^{-1}$. Cada unidade experimental contou com três linhas de oito plantas, no espaçamento de 3,0 $\mathrm{m}$ entrelinhas e 1,7 m entre plantas e ocupou uma área de $122,4 \mathrm{~m}^{2}$. Utilizou-se a linha central como área útil da parcela, excluindo-se a primeira e última planta da linha.

Após aração e gradagem fez-se a distribuição do calcário dolomítico em área total e a incorporação com grade, 50 dias antes do transplantio e em quantidade para elevar a saturação por bases a 70\% (RAIJ et al., 1996). Após esse período, procedeu-se a abertura dos sulcos com aproximadamente $30 \mathrm{~cm}$ de profundidade e realizou-se a adubação.

$\mathrm{O} \mathrm{N}$ foi aplicado na forma de nitrato de amônio em doses equivalentes a $30 \mathrm{~kg} \mathrm{~N}^{-1}$ no sulco e $90 \mathrm{~kg}$ $\mathrm{N}$ ha $^{-1}$ parcelado em três vezes. Para as doses de 50; 100; 200 e $300 \mathrm{~kg} \mathrm{~K}_{2} \mathrm{O} \mathrm{ha}{ }^{-1}$ fornecidas por meio de nitrato de potássio, foram necessários 26,$3 ; 22,6 ; 15,2$ e $7,8 \mathrm{~kg} \mathrm{~N} \mathrm{ha}^{-1}$ na forma de nitrato de amônio para complementar a dose de $30 \mathrm{~kg} \mathrm{~N}^{-1}$ prevista para o plantio e em cada uma das três coberturas.

No sulco, foram aplicados também $240 \mathrm{~kg} \mathrm{ha}^{-1}$ de $\mathrm{P}_{2} \mathrm{O}_{5}$ nas formas de superfosfato simples e de superfosfato triplo, para fornecer $50 \mathrm{~kg} \mathrm{ha}^{-1}$ de enxofre. Os tratamentos com dose de 200 e $300 \mathrm{~kg} \mathrm{~K}_{2} \mathrm{O} \mathrm{ha}^{-1}$ na fonte de $\mathrm{K}_{2} \mathrm{SO}_{4}$ receberam 67 e $100 \mathrm{~kg} \mathrm{~S} \mathrm{ha}^{-1}$.

As doses de $\mathrm{K}$ foram parceladas em $25 \%$ no sulco, $25 \%$ aos 7 dias após o transplantio (DAT), $25 \%$ aos 21 DAT e $25 \%$ aos 35 DAT, juntamente com o nitrogênio.

O híbrido utilizado foi Tide, que se caracteriza pela precocidade, apresentando ciclo médio do plantio à colheita de 80 a 90 dias, com frutos de formato redondo-ovalado e peso médio de 10 a $12 \mathrm{~kg}$, casca verdeescura com estrias verde-claras e polpa vermelhobrilhante. A semeadura foi realizada em bandejas de poliestireno expandido para 128 mudas, preenchidas com substrato comercial, as quais permaneceram em casa-devegetação por um período de 30 dias até o transplantio.

No primeiro experimento, o transplantio foi realizado em 03/10/2001 e, no segundo, em 14/02/2002, quando as plântulas apresentavam duas folhas definitivas. A partir dos 20 DAT, foram feitas adubações foliares semanalmente, junto com os defensivos, empregando 200 $\mathrm{mL}$ por $100 \mathrm{~L}$ de solução: $0,6 \mathrm{~g} \mathrm{~L}^{-1}$ de $\mathrm{Mg} ; 0,8 \mathrm{~g} \mathrm{~L}^{-1}$ de 
Ca e $0,05 \mathrm{~g} \mathrm{~L}^{-1}$ de B; 0,3 $\mathrm{g} \mathrm{L}^{-1}$ de $\mathrm{Zn} ; 0,2 \mathrm{~g} \mathrm{~L}^{-1}$ de $\mathrm{Mn}$ e $0,01 \mathrm{~g} \mathrm{~L}^{-1}$ de Mo. A pluviometria foi de $436 \mathrm{~mm}$ e 253 $\mathrm{mm}$, respectivamente, nos experimentos conduzidos de outubro a dezembro de 2001 e fevereiro a abril de 2002. Não foi realizada irrigação complementar, pois nas áreas experimentais, não existiam fontes de captação de água.

Além das pulverizações com defensivos agrícolas, foram realizadas capinas e penteamento das ramas. A colheita dos frutos foi iniciada no primeiro experimento aos 70 DAT e, no segundo, aos 65 DAT, sendo realizadas, respectivamente, três e duas colheitas com intervalo de sete dias. As características avaliadas foram: peso médio de fruto $(\mathrm{kg})$, número de frutos e produção por planta $(\mathrm{kg} /$ planta) e produtividade $\left(\mathrm{t} \mathrm{ha}^{-1}\right)$.

As análises de variância das características avaliadas foram realizadas isoladamente para cada experimento. Depois procedeu-se à análise conjunta dos experimentos com o auxílio do software SAS. Conforme Gomes (1990), para o fator quantitativo (doses de K) foi feita análise de regressão, ao passo que, para o fator qualitativo (fontes de K), foi utilizado o teste de Tukey ao nível de 5\% de probabilidade.

\section{RESULTADOS E DISCUSSÃO}

Verificou-se efeito significativo da época de cultivo para todas as características avaliadas. Para o número de frutos, produção por planta e produtividade, foi também significativa a interação fontes e doses de K e, para o peso médio de frutos, a interação época de cultivo e fontes de potássio.

No experimento conduzido de outubro a dezembro de 2001, a maior pluviosidade ocorrida ao longo do ciclo da cultura proporcionou maior peso médio e produção de frutos por planta e total. Como a significativa redução na quantidade de chuvas no cultivo de fevereiro a abril de 2002 ocorreu no terço final do ciclo cultural, correspondendo aos estádios de crescimento e maturação dos frutos, o número de frutos por planta não foi afetado, sendo ligeiramente superior ao da época anterior (Tabela 1).

Pode-se inferir que a menor produção por planta verificada no cultivo de fevereiro a abril de 2002 foi ocasionada pela redução no peso médio do fruto, motivada pela menor precipitação. O período de crescimento dos frutos é considerado por Marouelli et al. (1996) como o de maior exigência de água pela cultura. Segundo Grangeiro e Cecílio Filho (2002), nessa fase ocorrem os maiores acúmulos de massa seca e nutrientes pela melancieira, devidos à forte demanda exercida pelos frutos. O estresse hídrico nessa fase é altamente prejudicial, pois acelera a respiração, aumenta a atividade das enzimas hidrolíticas e reduz a intensidade de fotossíntese. Com isso, a translocação de carboidratos e outros compostos para os frutos é reduzida, com prejuízo no rendimento da cultura (MALAVOLTA e CROCOMO, 1982).

Além do comprometimento do metabolismo da melancieira nessas condições, a deficiência hídrica no solo reduz a absorção de nutrientes, resultante do menos fluxo de massa causada pela redução no fluxo transpiratório e do volume da solução de íons no solo; menos difusão iônica pela redução da mobilidade destes e menor interceptação radicular pela redução do crescimento e da densidade radicular (MENGEL e KIRKBY, 1987; POWER, 1990; MARSCHNER, 1995).

TABELA 1 - Número, peso médio, produção e produtividade de frutos de melancia, híbrido Tide, em função da época de cultivo. Borborema -SP.

\begin{tabular}{ccccc}
\hline Época* & $\begin{array}{c}\mathbf{N}^{\mathbf{0}} \text { de frutos } \\
\text { por planta }\end{array}$ & $\begin{array}{c}\text { Peso médio fruto } \\
\mathbf{( k g})\end{array}$ & $\begin{array}{c}\text { Produção de frutos por } \\
\text { planta }(\mathbf{k g})\end{array}$ & $\begin{array}{c}\text { Produtividade } \\
\left(\mathbf{t} \mathbf{~ h a}^{-\mathbf{1}} \mathbf{)}\right.\end{array}$ \\
\hline 1 & $1,6 \mathrm{~b}$ & $9,8 \mathrm{a}$ & $15,9 \mathrm{a}$ & $34,6 \mathrm{a}$ \\
2 & $1,8 \mathrm{a}$ & $7,0 \mathrm{~b}$ & $12,2 \mathrm{~b}$ & $24,3 \mathrm{~b}$ \\
\hline
\end{tabular}

\footnotetext{
${ }^{1}$ Médias seguidas de mesmas letras na coluna não diferem entre si pelo teste de Tukey a $5 \%$.

*Época 1: experimento conduzido de outubro a dezembro de 2001

Época 2: experimento conduzido de fevereiro a abril de 2002.
} 
O desdobramento da interação doses dentro de cada fonte de potássio para a característica número de frutos por planta revelou efeito significativo para as fontes $\mathrm{KCl}$ e $\mathrm{KNO}_{3}$. Nessas fontes, o maior número de frutos por planta estimado foi semelhante $(1,9)$; entretanto, as doses que proporcionaram esse maior número foram de 190 e $300 \mathrm{~kg} \mathrm{~K}_{2} \mathrm{O} \mathrm{ha}{ }^{-1}$, respectivamente para $\mathrm{KNO}_{3}$ e $\mathrm{KCl}$ (Figura 1). Embora o número de frutos tenha sido igual, foram necessários $110 \mathrm{~kg} \mathrm{~K}_{2} \mathrm{O} \mathrm{ha}^{-1}$ a mais, quando se usou a fonte $\mathrm{KCl}$ em relação ao $\mathrm{KNO}_{3}$, representando acréscimo de $58 \%$ na dose de $\mathrm{K}_{2} \mathrm{O}$ a ser aplicada. Para o $\mathrm{K}_{2} \mathrm{SO}_{4}$, o número médio de frutos foi de 1,7 (Figura 1).

De acordo com Mengel e Viro (1974), embora o potássio possa ser favorável ao incremento no peso médio do fruto, o efeito positivo dele é percebido, principalmente no aumento do número de frutos por planta. Quando a absorção de K, juntamente com outros nutrientes, é insuficiente, pode haver o retardamento na abertura floral e aumento da incidência de abortos (ATHERTON e HARRIS, 1986). No tomateiro, segundo Filgueira (2000), a adubação potássica, além de aprimorar a qualidade, evita a queda de frutos em formação, em razão de pedúnculos mais resistentes, aumentando, conseqüentemente, o número de frutos por planta.
Em meloeiro, Costa (2002) verificou tendência de acréscimo na fixação de frutos com aumento na concentração de potássio na solução nutritiva. Nerson et al. (1997), quando a cultura do meloeiro estava em deficiência de P e K, observaram uma inibição no crescimento vegetativo e redução na produção devidas à diminuição na fixação e no tamanho de frutos.

No cultivo de outubro a dezembro de 2001, não houve diferença significativa entre as fontes de potássio para o peso médio de fruto, o qual foi de $9,8 \mathrm{~kg}$, bem próximo daquele classificado por Villa et al. (2001) como especial $(>10 \mathrm{~kg})$ e que apresenta maior cotação comercial. No cultivo de fevereiro a abril de 2002, as fontes $\mathrm{KCl}$ e $\mathrm{KNO}_{3}$ não apresentaram diferenças significativas; entretanto, foram superiores ao $\mathrm{K}_{2} \mathrm{SO}_{4}$ (Tabela 2). Aumentos no peso médio de fruto ocasionados pela adubação potássica também foram verificados em melão (KANO, 2002) e tomate (PUJOS e MORAED, 1997). Esse aumento, segundo os autores, é atribuído ao papel importante que esse nutriente desempenha na translocação de fotossintatos das folhas para os frutos. Não obstante, plantas bem supridas em potássio têm a concentração de K elevada nos tecidos e, conseqüente redução do potencial hídrico, o que leva a um maior acúmulo de água nos tecidos (MONTOYA et al., 2002).

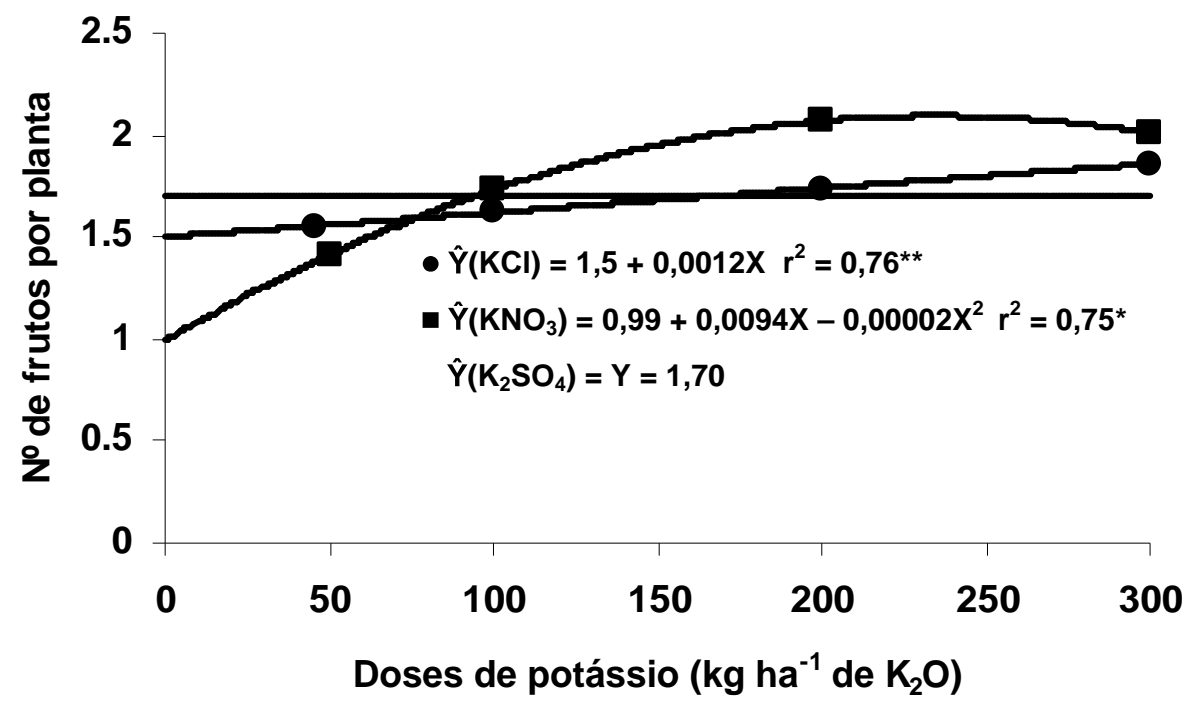

FIGURA 1 - Número de frutos por planta de melancia, híbrido Tide, em função das fontes e doses de potássio. Borborema - SP. 
TABELA 2 - Peso médio de frutos de melancia, híbrido Tide, em função de fontes de potássio e época de cultivo. Borborema - SP.

\begin{tabular}{ccc}
\hline \multirow{2}{*}{$\begin{array}{c}\text { Fontes de potás- } \\
\text { sio }\end{array}$} & \multicolumn{2}{c}{ Peso médio de frutos (kg) } \\
\cline { 2 - 3 } & Época $\mathbf{1}^{*}$ & Época 2 \\
\hline $\mathrm{KCl}$ & $9,5 \mathrm{a}^{1}$ & $7,2 \mathrm{a}$ \\
$\mathrm{KNO}_{3}$ & $9,9 \mathrm{a}$ & $7,1 \mathrm{a}$ \\
$\mathrm{K}_{2} \mathrm{SO}_{4}$ & $10,0 \mathrm{a}$ & $6,5 \mathrm{~b}$ \\
\hline
\end{tabular}

${ }^{1}$ Médias seguidas de mesmas letras na coluna não diferem entre si pelo teste de Tukey a $5 \%$.

*Época 1: experimento conduzido de outubro a dezembro de 2001

Época 2: experimento conduzido de fevereiro a abril de 2002.

A resposta diferenciada das fontes de potássio em função da época de cultivo foi provavelmente devida à diferença de pluviometria entre os experimentos. $\mathrm{O}$ maior volume e melhor distribuição das chuvas no período de outubro a dezembro proporcionaram umidade de solo satisfatória para a absorção de $\mathrm{K}$, independente do íon acompanhante.

No período seguinte, o estresse hídrico ocorrido no estádio pós frutificação prejudicou o rendimento da cultura. Segundo Fixen (1993), as melhores respostas das culturas em condições de estresse hídrico com aplicação de fertilizantes contendo $\mathrm{Cl}$ devem-se ao menor potencial osmótico causado pela absorção de $\mathrm{Cl}^{-}$pela planta, resultando em aumento do turgor dessa. Em tomate, a aplicação de $\mathrm{KCl}$ aumentou a eficiência de uso da água em condições de estresse hídrico (WILLIAMS e KRETCHMAN, 1990). Em beterraba, a utilização de fertilizantes com $\mathrm{Cl}$ manteve a turgescência e retardou a senescência das folhas, permitindo continuar a acumulação de açúcar por um período maior, proporcionando produtividades mais elevadas (ZEHLER et al., 1986).

O desdobramento da interação doses de potássio dentro de cada fonte para a produção por planta revelou efeito quadrático para $\mathrm{KCl}$ e $\mathrm{KNO}_{3}$ e cúbico para $\mathrm{K}_{2} \mathrm{SO}_{4}$ (Figura 2). A maior produção estimada por planta $(16,1 \mathrm{~kg})$ foi obtida com a aplicação de $111 \mathrm{~kg} \mathrm{~K} \mathrm{~K}_{2} \mathrm{O}$ $\mathrm{ha}^{-1}$ na forma de $\mathrm{K}_{2} \mathrm{SO}_{4}$, seguida pelo $\mathrm{KNO}_{3}(15,8 \mathrm{~kg}$ por planta) com a aplicação de $189 \mathrm{~kg} \mathrm{~K}_{2} \mathrm{O} \mathrm{ha}{ }^{-1}$ e pelo $\mathrm{KCl}(15,7 \mathrm{~kg}$ por planta) com a aplicação de $206 \mathrm{~kg}$ $\mathrm{K}_{2} \mathrm{O}$ ha $^{-1}$. O $\mathrm{KNO}_{3}$ proporcionou maior incremento na produção de frutos por planta $(33 \%)$, em relação ao $\mathrm{KCl}$
(23\%) e $\mathrm{K}_{2} \mathrm{SO}_{4}(17 \%)$, comparando-se os valores obtidos com a aplicação de $50 \mathrm{~kg} \mathrm{~K}_{2} \mathrm{O}$ ha $^{-1}$ com aquele que favoreceu a produção máxima. Aumentos significativos na produção de melancia em função da adubação potássica também foram observados por Sundstrom e Carter (1983), Deswal e Patil (1984), Zeng e Jiang (1989), Simonne et al. (1992) e Locascio e Hochmuth (2002).

A produtividade em função das doses de potássio apresentou comportamento quadrático em todas as fontes, com pontos de máximos atingidos nas doses de 132; 193 e $205 \mathrm{~kg} \mathrm{~K}_{2} \mathrm{O} \mathrm{ha}{ }^{-1}$, respectivamente, para $\mathrm{K}_{2} \mathrm{SO}_{4}$, $\mathrm{KNO}_{3}$ e $\mathrm{KCl}$, sendo as respectivas produtividades de 32,4; 34,4 e 32,4 t ha ${ }^{-1}$ (Figura 3). Observa-se que as produtividades máximas obtidas com as fontes $\mathrm{KCl}$ e $\mathrm{K}_{2} \mathrm{SO}_{4}$ foram semelhantes; entretanto, o $\mathrm{KNO}_{3}$ foi ligeiramente superior às demais. Por outro lado, a diferença nas doses foram significativas, sendo necessária a aplicação de 73 e $61 \mathrm{~kg} \mathrm{~K}_{2} \mathrm{O}$ ha $^{-1}$ a mais, como $\mathrm{KCl}$ e $\mathrm{KNO}_{3}$, para se atingir a produtividade máxima, em relação ao $\mathrm{K}_{2} \mathrm{SO}_{4}$, representando acréscimos de 55 e $46 \%$, respectivamente.

Do ponto de vista de eficiência, verifica-se que o $\mathrm{K}_{2} \mathrm{SO}_{4}$ foi superior às demais, pois para cada $\mathrm{kg}$ de $\mathrm{K}_{2} \mathrm{O}$ utilizado na forma desse fertilizante, obteve-se uma produtividade de $245 \mathrm{~kg} \mathrm{ha}^{-1}$, superior aos 158 e $178 \mathrm{~kg}$ $\mathrm{ha}^{-1}$ obtidos, respectivamente, com $\mathrm{KCl}$ e $\mathrm{KNO}_{3}$.

Considerando-se as equações da Figura 3, foram estimadas as produções e as doses das fontes de potássio correspondentes a $90 \%$ da máxima. As doses de cada fonte correspondentes a $90 \%$ da máxima produtividade proporcionaram reduções de 48; 54 e $72 \%$ das doses de $\mathrm{KNO}_{3}, \mathrm{KCl}$ e $\mathrm{K}_{2} \mathrm{SO}_{4}$, respectivamente (Tabela 3). Essas grandes reduções nas doses dos fertilizantes são acompanhadas de reduções de apenas $10 \%$ na produtividade máxima, representando, assim, uma considerável economia com a adubação potássica e levando certamente a uma relação benefício/custo substancialmente maior.

Um dos fatores que pode ter contribuído para maior eficiência do $\mathrm{K}_{2} \mathrm{SO}_{4}$ em relação às demais fontes é que o ânion $\mathrm{SO}_{4}{ }^{2-}$, em relação ao $\mathrm{Cl}^{-}$e $\mathrm{NO}_{3}{ }^{-}$, é menos lixiviado no solo e há maior possibilidade de adsorção. Com isso, as perdas de $\mathrm{K}^{+}$por lixiviação, aplicado na forma de $\mathrm{K}_{2} \mathrm{SO}_{4}$, podem ser minimizadas, aumentando assim a eficiência desse. Saurat e Boulay (1985), citados por Nogueira et al. (2001), comentam que há duas razões pelas quais o ânion $\mathrm{Cl}^{-}$é lixiviado mais rapidamente do que o $\mathrm{SO}_{4}{ }^{2-}$ : (1) o ânion $\mathrm{Cl}^{-}$tem maior tamanho iônico, sendo fracamente adsorvido; (2) o íon $\mathrm{Cl}^{-}$, sendo monovalente, é mais rapidamente hidratável na solução do que o íon $\mathrm{SO}_{4}{ }^{2-}$. Conseqüentemente, o $\mathrm{K}$ na 
forma de fertilizante $\mathrm{KCl}$ é mais rapidamente lixiviado do que seria na forma de fertilizante $\mathrm{K}_{2} \mathrm{SO}_{4}$.

No tomateiro, a produtividade comercial foi $19 \%$ maior com a aplicação de $\mathrm{KNO}_{3}$ em relação ao $\mathrm{KCl}$, mas não diferiu significativamente do $\mathrm{K}_{2} \mathrm{SO}_{4}$. Esse resultado foi verificado por Locascio et al. (1997) em apenas um experimento de uma série de nove. Em trabalhos anteriores, quando foram comparadas as fontes $\mathrm{KNO}_{3} \mathrm{e}$
$\mathrm{KCl}$, as produtividades de tomate não diferiram significativamente entre as fontes (LOCASCIO et al., 1982). Quando os fertilizantes avaliados foram $\mathrm{K}_{2} \mathrm{SO}_{4}$ e $\mathrm{KCl}$, não foi observada diferença de produtividade na cultura do tomateiro (LOCASCIO et al., 1990) mas, nas culturas de berinjela (WUZHONG, 2002) e de batata (WESTERMANN et al., 1994), o $\mathrm{K}_{2} \mathrm{SO}_{4}$ proporcionou rendimentos superiores aos obtidos com $\mathrm{KCl}$.

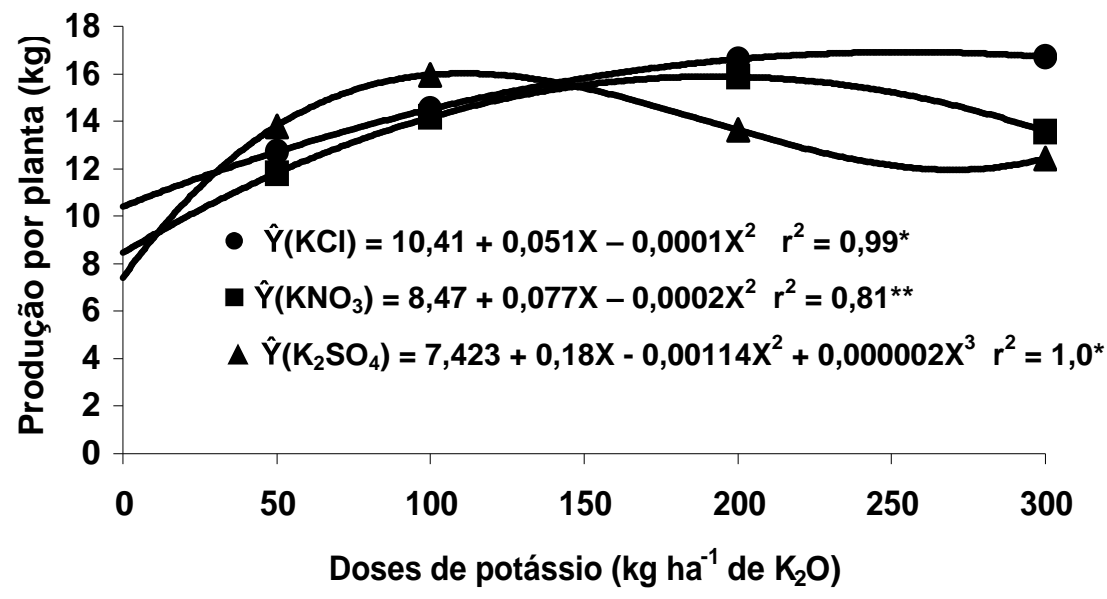

FIGURA 2 - Produção de frutos por planta de melancia, híbrido Tide, em função das doses e fontes de potássio. Borborema - SP.

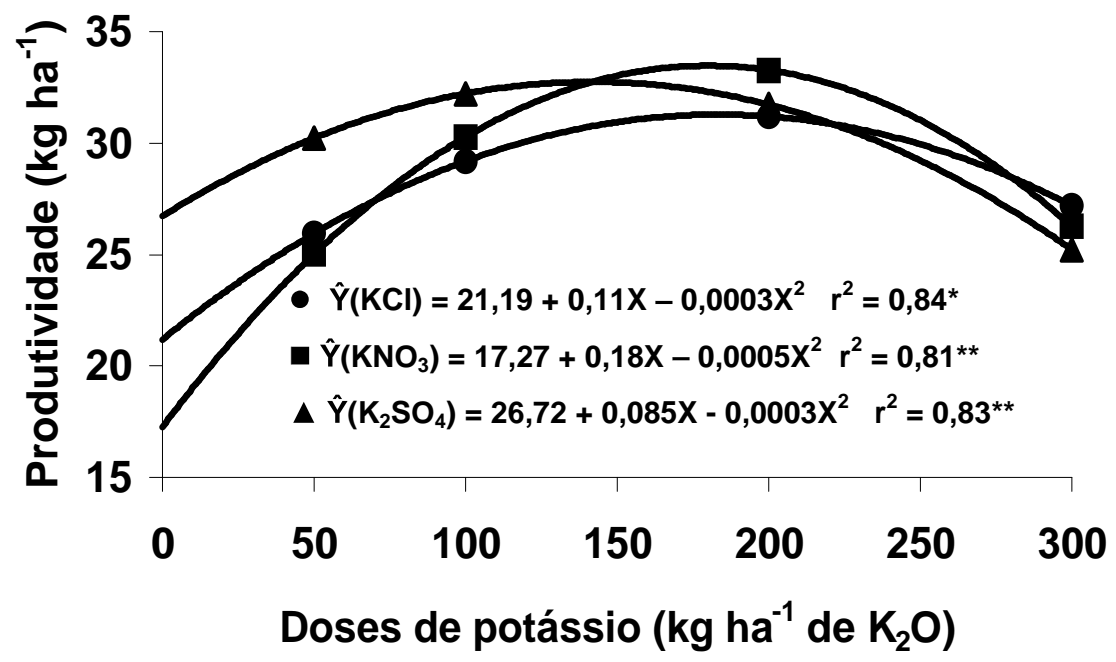

FIGURA 3 - Produtividade de frutos de melancia, híbrido Tide, em função das doses e fontes de potássio. Borborema $-\mathrm{SP}$. 
TABELA 3 - Doses de potássio estimada para obter a máxima produtividade da cultura da melancia e $90 \%$ dessa, com os fertilizantes $\mathrm{KCl}, \mathrm{KNO}_{3}$ e $\mathrm{K}_{2} \mathrm{SO}_{4}$. Borborema - SP.

\begin{tabular}{|c|c|c|c|c|}
\hline \multirow{2}{*}{ Fontes de potássio } & \multicolumn{2}{|c|}{ Produtividade $\left(\mathrm{t} \mathrm{ha}^{-1}\right)$} & \multicolumn{2}{|c|}{ Dose $\left(\mathrm{kg} \mathrm{K}_{2} \mathrm{O} \mathrm{ha}^{-1}\right)$} \\
\hline & Máxima & $90 \%$ & Máxima & $90 \%$ \\
\hline $\mathrm{KCl}$ & 32,4 & 29,2 & 205 & 95 \\
\hline $\mathrm{KNO}_{3}$ & 34,4 & 31,0 & 193 & 100 \\
\hline $\mathrm{K}_{2} \mathrm{SO}_{4}$ & 32,4 & 29,2 & 132 & 34 \\
\hline
\end{tabular}

Tomando-se por base a dose recomendável de cada fonte de potássio $(90 \%$ da produção máxima estimada), verifica-se, para as condições desta pesquisa, uma quantidade inferior àquela recomendada por Raij et al. (1996) para o Estado de São Paulo, que é de $190 \mathrm{~kg}$ de $\mathrm{K}_{2} \mathrm{O}$ ha $^{-1}$.

No cultivo de outubro a dezembro de 2001 , foi obtida uma produtividade média de $34,6 \mathrm{t} \mathrm{ha}^{-1}$, superior as 24,3 $\mathrm{t} \mathrm{ha}^{-1}$ do cultivo de fevereiro a abril de 2002 (Tabela 1). No plantio de melancia no Estado de São Paulo, realizado no período de janeiro a fevereiro, cultivo esse chamado de "safrinha", embora são conseguidos melhores preços pelo produto, a produtividade dos frutos é inferior à da safra normal, a qual corresponde ao plantio realizado de julho a setembro, em razão principalmente da melhor distribuição das chuvas nesse período e do atendimento à demanda da planta.

\section{CONCLUSÕES}

a) O maior número de frutos $(1,9)$ foi obtido nas

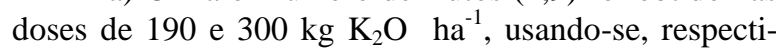
vamente, como fontes $\mathrm{KNO}_{3}$ e $\mathrm{KCl}$. Não houve diferença significativa entre as doses para $\mathrm{K}_{2} \mathrm{SO}_{4}$.

b) No cultivo de fevereiro a abril de 2002 , o $\mathrm{KCl}$ proporcionou maior peso médio de fruto.

c) A maior produção por planta foi obtida com $\mathrm{K}_{2} \mathrm{SO}_{4}$, seguido por $\mathrm{KNO}_{3}$ e $\mathrm{KCl}$, nas doses de $111 ; 189$ e $206 \mathrm{~kg} \mathrm{~K}_{2} \mathrm{O}$ ha $^{-1}$, respectivamente.

d) As maiores produtividades foram obtidas com aplicação de 132, 193 e $205 \mathrm{~kg} \mathrm{~K}_{2} \mathrm{O}$ ha $^{-1}$, utilizando-se como fontes $\mathrm{K}_{2} \mathrm{SO}_{4}, \mathrm{KNO}_{3}$ e $\mathrm{KCl}$, respectivamente.

e) As doses de potássio, que proporcionaram $90 \%$ da produtividade máxima, reduziram em $74 \%$, $54 \%$ e $48 \%$ as doses de $\mathrm{K}_{2} \mathrm{SO}_{4}, \mathrm{KCl}$ e $\mathrm{KNO}_{3}$, necessárias para obter as produtividades máximas.

f) O cultivo de outubro a dezembro de 2001 proporcionou maior peso médio, produção de frutos por planta e produtividade, ao passo que o maior número de frutos por planta foi obtido no cultivo de fevereiro a abril de 2002.

\section{AGRADECIMENTOS}

À FAPESP, por auxílio financeiro concedido (processo 2000/01797-0), aos produtores rurais Colombo e João Palhari, e a Alécio Schiavon Júnior, da Empresa Syngenta Seeds.

\section{REFERÊNCIAS BIBLIOGRÁFICAS}

ATHERTON, J. G.; HARRIS, G. P. Flowering. In: ATHERTON, J. G.; RUDICH, J. (Eds.). The tomato crop. New York: Chapman and Hall, 1986. p. 167200.

COSTA, C. C. Concentração de potássio na solução nutritiva e números de frutos por planta sobre a produção e qualidade dos frutos do meloeiro. 2002. 51 f. Dissertação (Mestrado em Agronomia) - Faculdade de Ciências Agrária e Veterinárias, Universidade Estadual Paulista, Jaboticabal, 2002.

DESWAL, I. S.; PATIL, V. K. Effects of N, P and K on the fruit of water melon. Journal of Maharashtra Agricultural Universities, [S.1.], v. 9, n. 3, p. 308-309, 1984.

EMPRESA BRASILEIRA DE PESQUISA AGROPECUÁRIA. Sistema brasileiro de classificação de solos. Brasília, 1999. 412 p.

FAQUIN, V. Nutrição mineral de plantas. Lavras: ESAL-FAEPE, 1994. 227 p.

FILGUEIRA, F. A. R. Novo manual de olericultura: agrotecnologia moderna na produção e comercialização de hortaliças. Viçosa: UFV, 2000. 234 p. 
FIXEN, P. E. Crop responses to chloride. Advances in Agronomy, San Diego, v. 50, p. 107-150, 1993.

GOMES, F. P. Curso de estatística experimental. 12. ed. Piracicaba: Nobel, 1990. 466 p.

GRANGEIRO, L. C.; CECÍlLIO FILHO, A. B. Marcha de absorção de nutrientes pela cultura da melancia. In: REUNIÃO BRASILEIRA DE FERTILIDADE DO SOLO E NUTRIÇÃO DE PLANTAS, 25.; REUNIÃO BRASILEIRA SOBRE MICORRIZAS, 9.; SIMPÓSIO BRASILEIRO DE MICROBIOLOGIA DO SOLO, 7.; REUNIÃO BRASILEIRA DE BIOLOGIA DO SOLO, 4., 2002, Rio de Janeiro. Anais... Rio de Janeiro: [s.n.], 2002. 1 CD-ROM.

KANO, C. Extrações de nutrientes pelo meloeiro rendilhado cultivado em ambiente protegido com a adição de potássio e $\mathbf{C O}_{2}$ na água de irrigação. 2002. 102 f. Dissertação (Mestrado em Agronomia) - Escola Superior de Agricultura Luiz de Queiroz, Universidade de São Paulo, Piracicaba, 2002.

LOCASCIO, S. J.; HOCHMUTH, G. J. Watermelon production as influenced by lime, gypsum, and potassium. HortScience, Alexandria, v. 37, n. 2, p. 322-324, 2002.

LOCASCIO, S. J.; HOCHMUTH, G. J.; OLSON, S. M.; HOCHMUTH, R. C.; CSIZINSZKY, A. A.; SHULER, K. D. Potassium source and rate for polythylene-mulched tomatoes. HortScience, Alexandria, v. 32, n. 7, p. 1204-1207, 1997.

LOCASCIO, S. J.; MYERS, J. M.; FISKELL, J. G. A. Nitrogen apllication timing and source for drip irrigated tomatoes. Warwick: Proc. Ninth Intl. Plant Nutr. Colloq., 1982. 328 p.

LOCASCIO, S. J.; OLSON, S. M.; GULL, D. D. Potassium source and rate and calcium rate effects on tomato yield and quality. HortScience, Alexandria, v. 25, n. 9, p. 1129, 1990.

MALAVOLTA, E.; CROCOMO, O. J. Funções do potássio nas plantas. In: YAMADA, T.; IGUE, K.; MUZILLI, O.; USHERWOOD, N. R. Potássio na agricultura brasileira. Piracicaba: POTAFOS, 1982. p. 95-162.

MAROUELli, W. A.; SILVA, W. L. C.; SILVA, H. R. Manejo da irrigação em hortaliças. Brasília: EMBRAPA-CNPH, 1996. 72 p.
MARSCHNER, H. Mineral nutrition of higher plants. San Diego: Academic, 1995. 889 p.

MAYNARD, E. Potassium source and rate effects on muskmelon yield and fruit quality. Disponível em: 4http://Www.ppi-far.org

MENGEL, K.; KIRKBY, E. A. Copper, further elements of importance. In: MENGEL, K.; KIRKBY, E. A. Principles of plant nutrition. 4. ed. Berne: International Potash Institute, 1987. p. 537-588.

MENGEL, K.; VIRO, M. Effect of potassium supply on the transport of photosynthates to the fruits of tomatoes (Lycopersicon esculentum). Physiology Plant, Minneapolis, v. 30, p. 295-300, 1974.

MONTOYA, R. B.; SPINOIA, A. G.; GARCIA, P. S.; PAREDES, D. G. Demanda de potasio del tomate tipo sadette. Disponivel em: ${ }^{1} \leq \mathrm{h}$ ttp://Www.chapingd. $\mathrm{mx} /$ terra/contenido/20/4art 391-399.pdf. $>$. Acesso em: 18 dez. 2002.

NERSON, H.; EDELSTEIN, M.; BERDUGO, R.; ANKORION, Y. Monopotassium phosphate as a phosphorus and potassium source for greenhousewinter-grown cucumber and muskmelon. Journal of Plant Nutrition, Monticello, v. 20, n. 2/3, p. 335-344, 1997.

NOGUEIRA, F. D.; SILVA, E. de B.; GUIMARÃES, P. T. G. Adubação potássica do cafeeiro: sulfato de potássio. Washington: SOPIB, 2001. 81 p.

PANIQUE, E.; KELLING, K. A.; SCHULTE, E. E.; HERO, D. E.; STEVENSON, W. R.; JAMES, R. V. Potassium rate and source effects on potato yield, quality, and disease interaction. American Potato Journal, Orono, v. 74, p. 379-398, 1997.

POWER, J. F. Role of moisture stress in plant nutritional functions. In: BALIGAR, V. C.; DUNCAN, R. R. Crops as enhancers of nutrient use. San Diego: Academic, 1990. p. 453-474.

PUJOS, A.; MORAED, P. Effects of potassium deficiency on tomato growth and mineral nutrition at the early production stage. Plant Soil, [S.1.], v. 189, p. 189-196, 1997.

RAIJ, B. V.; CANTARELLA, H.; QUAGGIO, J. A.; FURLANI, A. M. C. Recomendações de adubação e calagem para o estado de São Paulo. Campinas: [s.n.], 1996. 285 p. (Boletim Técnico, 100). 
SIMONNE, E. H.; MILLS, H. A.; SMITTLE, D. A. Ammonium reduces growth fruit yield and fruit quality of watermelon. Journal of Plant Nutrition, Monticello, v. 15, n. 12, p. 2727-2741, 1992.

SUNDSTROM, F. J.; CARTER, S. J. Influence of K and $\mathrm{Ca}$ on quality and yield of watermelon. Journal American Society for Horticultural Science, Alexandria, v. 108, n. 5, p. 879-881, 1983.

WESTERMANN, D. T.; TINDALL, T. A.; JAMES, D. W.; HURST, R. L. Nitrogen and potassium fertilization of potatoes: yield and specific gravity. American Potato Journal, Orono, v. 71, p. 417-431, 1994.

WILliAMS, J. D.; KRETCHMAN, D. W. Potassium chloride concentration during production affects tomato transplant response to postproduction waters stress. HortScience, Alexandria, v. 25, n. 9, p. 1129, 1990.
WUZHONG, N. Yield and quality of fruits of solanaceous crops as affected by potassium fertilization. Better Crops, Atlanta, v. 13, n. 1, p. 6$8,2002$.

VILLA, W.; GROPPO, G. A.; TESSARIOLI NETO, J.; GELMINI, G. A. Cultura da melancia. Campinas: [s.n.], 2001. 52 p. (Boletim Técnico, 243).

ZEHLER, E.; KREIP, H.; GETHING, P. A. Sulfato de potássio e cloreto de potássio: sua influência na produção e na qualidade das plantas cultivadas. Campinas: Fundação Cargill, 1986. 111 p.

ZENG, Q. Y.; JIANG, X. L. Influence of potash fertilizers containing chlorine on the quality of watermelon. Soils, Longjumeau, v. 20, n. 3, p. 144$146,1988$. 